\title{
Comparative Analysis of Diffusional Kurtosis Imaging, Diffusion Tensor Imaging, and Diffusion-Weighted Imaging in Grading and Assessing Cellular Proliferation of Meningiomas
}

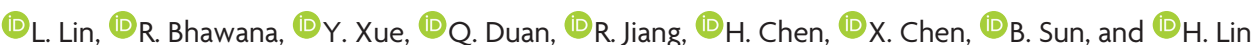

\begin{abstract}
BACKGROUND AND PURPOSE: An accurate evaluation of the World Health Organization grade and cellular proliferation is particularly important in meningiomas. Our aim was to prospectively evaluate and compare diffusional kurtosis imaging, DTI, and DWI metrics in determining the grade and cellular proliferation of meningiomas.
\end{abstract}

MATERIALS AND METHODS: Ninety-six consecutive patients with histopathologically confirmed meningiomas were included in this study. Mean kurtosis, radial kurtosis, axial kurtosis, fractional anisotropy, mean diffusivity, and ADC were semiautomatically obtained in the solid components of tumors. Each normalized diffusion value was compared between high-grade meningiomas and low-grade meningiomas using the Mann-Whitney $U$ test. Receiver operating characteristic, multiple logistic regression, and Pearson correlation analysis were used for statistical evaluations.

RESULTS: Diffusional kurtosis imaging metrics (mean kurtosis, radial kurtosis, and axial kurtosis) were significantly higher in high-grade meningiomas than in low-grade meningiomas $(P \leq .001)$. Mean diffusivity and ADC were significantly lower in high-grade meningiomas than in low-grade meningiomas $(P=.003$ and .002). Mean kurtosis had significantly greater area the under curve values than mean diffusivity and fractional anisotropy in differentiating high-grade meningiomas from low-grade meningiomas ( $P=.038$ and .002). Mean kurtosis was the only variable that could be used to independently differentiate high-grade meningiomas and low-grade meningiomas $(P<.001)$. Significant correlations were found between the Ki-67 labeling index and kurtosis metrics $(P<.001)$, as well as for mean diffusivity and ADC $(P=.004$, and .007).

CONCLUSIONS: Compared with other diffusion metrics, mean kurtosis may serve as an optimal parameter for evaluating and predicting the meningioma grade. Moreover, diffusion metrics may potentially reflect cellular proliferation.

ABBREVIATIONS: $A K=$ axial kurtosis; $D K I=$ diffusional kurtosis imaging; $F A=$ fractional anisotropy; $F S P G R=$ fast-spoiled gradient recalled; $H G M=$ high-grade meningioma; LGM = low-grade meningioma; $\mathrm{MD}=$ mean diffusivity; $\mathrm{MK}=$ mean kurtosis; $\mathrm{PPV}=$ positive predictive value; $\mathrm{RK}=$ radial kurtosis

M eningiomas are the most common type of intracranial brain tumors, accounting for approximately $30 \%$ of all primary brain neoplasms. ${ }^{1}$ Pathologically, meningiomas are classified into 3 grades according to the World Health Organization. ${ }^{2}$ The grad-

Received November 15, 2017; accepted after revision February 24, 2018.

From the Departments of Radiology (L.L., R.B., Y.X., Q.D., R.J., B.S., H.L.) and Pathology (H.C.), Fujian Medical University Union Hospital, Fuzhou, Fujian, China; and Department of Radiology (X.C.), Fujian Cancer Hospital and Fujian Medical University Cancer Hospital, Fuzhou, Fujian, China.

This work was supported by grants from the Youth Scientific Research Project of Fujian Provincial Health and the Family Planning Commission, China (No.2017-2-24) and the Natural Science Foundation of Fujian Province, China (No.2015J01396).

Please address correspondence to Yunjing Xue, MD, Department of Radiology, Fujian Medical University Union Hospital, No. 29 Xinquan Rd, Fuzhou, Fujian 350001, P.R. China; e-mail: xueyunjing@126.com

- Indicates open access to non-subscribers at www.ajnr.org

三Indicates article with supplemental on-line tables.

Indicates article with supplemental on-line photo.

http://dx.doi.org/10.3174/ajnr.A5662 ing of meningiomas has a high clinical relevance for determining treatment strategy and evaluating prognosis. However, the patient's prognosis can not only be determined by the tumor grade but also depends on the proliferative activity of tumors. ${ }^{3}$ Previous studies have documented that $\mathrm{Ki}-67$ expression is an important biomarker of cellular proliferation. ${ }^{4}$ Therefore, an evaluation of Ki-67 expression is also required in clinical practice for meningiomas.

At present, the presurgical diagnosis of meningiomas mainly relies on their radiologic features. However, it is difficult to distinguish low-grade (World Health Organization grade I) from high-grade (World Health Organization grade II or III) meningiomas. Heterogeneous enhancement, unclear tumor-brain interface, marked perilesional edema, and parenchymal or bone invasion may be indicative of high-grade meningiomas (HGMs). ${ }^{5}$ However, no specific or reliable features of conventional MR imaging have been found to differentiate HGMs from low-grade meningiomas (LGMs). 
Diffusion MR imaging is a technique that can measure the degree of mobility of water molecules within biologic tissue. Conventional DWI and DTI can provide conventional diffusion metrics, such as ADC, fractional anisotropy (FA), and mean diffusivity (MD), but they are not sufficiently accurate in evaluating the meningioma grade and Ki-67 expression ${ }^{3,6-10}$ because conventional DWI and DTI assume that water diffusion follows a Gaussian distribution. However, the complexity of normal and pathologic tissue leads to hindered diffusion of water molecules and, therefore, should follow a nonGaussian distribution. ${ }^{11,12}$ Diffusional kurtosis imaging (DKI) is an advanced non-Gaussian diffusion imaging technique that can more accurately characterize the complicated water diffusion in pathologic tissues and provide additional information about tumor heterogeneity by measuring the kurtosis metrics, including mean kurtosis (MK), axial kurtosis (AK), and radial kurtosis (RK). ${ }^{13}$ In recent years, several studies have been conducted using DKI in the diagnosis and differential diagnosis of intracranial neoplasms, including glioma, metastasis, and lymphoma. ${ }^{11,14-16}$ However, to our knowledge, the use of DKI in evaluating meningiomas was still lacking, and no comparison of DKI with DTI and DWI was reported. Because DKI, DTI, and DWI may reflect microstructural features of tumors, it should be valuable to explore and compare their roles in the grading of meningiomas. Thus, the aim of this study was to evaluate and compare the utility of diffusion metrics obtained from DKI (MK, AK, RK), DTI (FA, MD), and DWI (ADC) in grading meningiomas and to assess the correlations between diffusion metrics and the Ki-67 labeling index.

\section{MATERIALS AND METHODS \\ Patients}

Studies were performed abiding by the rules and guidelines of the Fujian Medical University Union Hospital committee on clinical investigations. Informed consent from all the patients was obtained following the protocol set by the local ethics committee of the hospital. From our institution, 102 patients with suspected meningioma on conventional MR imaging between August 2014 and October 2016 were consecutively enrolled in the study. Preoperative imaging, including DWI and DKI, was performed, and image data were prospectively analyzed. The inclusion criteria were as follows: 1) histopathologically confirmed as meningiomas, and 2) surgical resection performed within 10 days after the MR imaging examination. We applied the following exclusion criteria: patients with any previous relevant treatment history (including radiation therapy, chemotherapy, or an operation) and MR imaging data with motion artifacts. Histologic diagnoses and tumor grading were based on the 2016 World Health Organization Classification of Tumors of the Central Nervous System. ${ }^{2}$ Three patients were excluded because of substantial motion artifacts, and 3 patients were excluded because their lesions were confirmed to be nonmeningiomas. Finally, 96 patients were included.

\section{Data Acquisition}

A 3T MR imaging scanner (Discovery 750 system; GE Healthcare, Milwaukee, Wisconsin) and an 8-channel receiver head coil were used to examine all the patients before the operation. Imaging data were acquired by 2 blinded neuroradiologists independently (with 6 and 17 years' experience).
All patients underwent conventional and contrast-enhanced MR imaging. MR imaging was performed using the following routine sequences: axial T1-weighted FLAIR images $(\mathrm{TR} / \mathrm{TE}=1750 / 23 \mathrm{~ms}$, $\mathrm{TI}=780 \mathrm{~ms}, \mathrm{NEX}=1$, matrix $=320 \times 320, \mathrm{FOV}=24 \mathrm{~cm}$, slice thickness $=5 \mathrm{~mm}$, spacing $=1.5 \mathrm{~mm}$ ); axial T2-weighted FSE images $(\mathrm{TR} / \mathrm{TE}=6488 / 94 \mathrm{~ms}, \mathrm{NEX}=1.5$, matrix $=512 \times 512, \mathrm{FOV}=$ $24 \mathrm{~cm}$, slice thickness $=5 \mathrm{~mm}$, spacing $=1.5 \mathrm{~mm}$ ); axial T2weighted FLAIR images $(\mathrm{TR} / \mathrm{TE}=8500 / 143 \mathrm{~ms}$, TI $=2100 \mathrm{~ms}$, flip angle $=111^{\circ}, \mathrm{NEX}=1$, matrix $=288 \times 224, \mathrm{FOV}=24 \mathrm{~cm}$, slice thickness $=5 \mathrm{~mm}$, spacing $=1.5 \mathrm{~mm}$ ); contrast-enhanced $3 \mathrm{D}$ axial T1-weighted fast-spoiled gradient recalled (FSPGR) images (TR/ $\mathrm{TE}=8.2 / 3.2 \mathrm{~ms}$, matrix $=256 \times 256$, slice thickness $=1 \mathrm{~mm}$, $\mathrm{FOV}=24 \mathrm{~cm}, \mathrm{TI}=450 \mathrm{~ms}$, flip angle $=12^{\circ}, 144$ contiguous partitions). Postcontrast images were obtained after an intravenous contrast injection of $0.1 \mathrm{mmol} / \mathrm{kg}$ of gadopentetate dimeglumine (Magnevist; Bayer HealthCare Pharmaceuticals, Wayne, New Jersey).

DKI used a spin-echo EPI diffusion sequence for image acquisition $(\mathrm{TR} / \mathrm{TE}=6000 / 94 \mathrm{~ms}, \mathrm{NEX}=1$, matrix $=128 \times 128$, number of sections $=48$, sections thickness $=3 \mathrm{~mm}$, spacing $=0 \mathrm{~mm}$, $\mathrm{FOV}=24 \mathrm{~cm}, \mathrm{~B}_{0}=3, b$ values $=1000$ and $2000 \mathrm{~s} / \mathrm{mm}$, number of directions $=30$ for each, acquisition time $=6$ minutes 24 seconds).

A spin-echo EPI DWI sequence was also used. Parameters were as follows: $\mathrm{TR} / \mathrm{TE}=3000 / 70 \mathrm{~ms}, \mathrm{NEX}=4$, matrix $=160 \times$ 160 , number of sections $=20$, sections thickness $=5 \mathrm{~mm}$, spacing $=1.5 \mathrm{~mm}, \mathrm{FOV}=24 \mathrm{~cm}, b=0$ and $1000 \mathrm{~s} / \mathrm{mm}^{2}$, and acquisition time $=42$ seconds.

All MR imaging was performed on the same slices paralleling the line combining the anterior/posterior commissure with the same range to cover the entire brain.

\section{Image Processing}

The DKI dataset was first corrected for eddy current distortion and simple head motion using the FMRIB Software Library (FSL; http:// www.fmrib.ox.ac.uk/fsl) in reference to the $\mathrm{B}_{0}$ images. All data were used to calculate the diffusion kurtosis $\left(b=0,1000\right.$, and $2000 \mathrm{~s} / \mathrm{mm}^{2}$ ) and diffusion tensor $\left(b=0\right.$ and $1000 \mathrm{~s} / \mathrm{mm}^{2}$ ) simultaneously. The DKI and DTI data were processed by using the Diffusional Kurtosis Estimator (Version 2.5.1; Medical University of South Carolina, Charleston, South Carolina). DWI was acquired in 3 orthogonal directions and combined into a trace image, and ADC maps were generated automatically by the MR imaging system software. The diffusion metric maps were processed using ImageJ software (Version 1.50i; National Institutes of Health, Bethesda, Maryland).

Before we delineated the ROI, the image resolution and number of slices of enhanced T1 FSPGR, T2 FSE, and ADC maps were changed to match the DKI metric maps. Although the scan matrix, slice thickness, and spacing were different in enhanced $\mathrm{T} 1$ FSPGR, T2 FSE, DWI, and DKI, the image resolutions finally generated by the scanner were $256 \times 256,512 \times 512,256 \times 256$, and $256 \times 256$, respectively, due to interpolation. Thus, the image resolution of T2 FSE was first resized to $256 \times 256$, and the number slices of enhanced T1 FSPGR, T2 FSE, and ADC was changed to 48 without interpolation. All these protocols were finished in ImageJ, as shown in On-line Figure.

We performed a semiautomatic ROI-setting method, in line with our previous study. ${ }^{17}$ Two neuroradiologists (with 7 and 10 years' 
experience) blinded to the cases and unaware of the histopathologic diagnosis randomly reviewed and analyzed image data, and the average values of the 2 readers were collected as the final results. According to conventional MR images, especially contrast-enhanced T1weighted images, ROIs over the solid tumor and contralateral normal-appearing white matter were semiautomatically delineated using the Wand Tool in ImageJ. Cystic components, necrosis, hemorrhage, and calcification were avoided while estimating the solid region of the tumor. Contralateral normal-appearing white matter was defined in the centrum semiovale in the hemisphere contralateral to the lesion in accordance with former studies. ${ }^{7,10}$ If a tumor was located on the midline, the ROI for comparison was chosen in the centrum semiovale of both hemispheres, and the mean value was used. Afterward, the same ROIs were obtained from anatomic MR images for all parametric maps. Furthermore, the diffusion values in the solid components of tumors were normalized to the corresponding values in the contralateral normal-appearing white matter to reduce intersubject variation.

\section{Pathology and Immunohistochemistry}

The pathologic and immunohistochemical diagnoses of the meningiomas were determined by a neuropathologist (with 7 years' experience). In each case, the tumor proliferation index was estimated as the percentage of tumor cell nuclei labeled with the Ki-67 monoclonal antibody in formalin-fixed paraffin tissue sections. All cells with nuclear staining of any intensity were considered positive for Ki-67; areas with the highest density of positive tumor nuclei were selected for counting.

\section{Statistical Analysis}

Statistical analyses were performed with SPSS software (Version 19.0. IBM, Armonk, New York) and MedCalc for Windows (Version 11.4.2.0; MedCalc Software, Mariakerke, Belgium). The demographic data, radiologic features, and normalized diffusion metrics were compared between HGM and LGM with the $\chi^{2}$ test for categoric variables, independent-sample $t$ tests for normally distributed continuous variables, or the Mann-Whitney $U$ test for other continuous variables. The receiver operating characteristic curve analyses were performed to determine the diagnostic efficiency of normalized diffusion parameters. The $Z$-test was applied to compare the differences in areas under the curve among all diffusion metrics. A stepwise multiple logistic regression analysis of all normalized diffusion metrics was also performed to find the most valuable metric for grading meningiomas. The associations between $\mathrm{Ki}-67$ values and normalized diffusion metrics were calculated with the Pearson correlation analyses. The interobserver variability of measurements was assessed using an intraclass correlation coefficient. $P$ values $<.05$ were considered significant for all the tests.

\section{RESULTS}

\section{Patient Characteristics and Tumor Features on Conventional MR Imaging}

Among all the patients, 84 (87.5\%) had LGMs and 12 (12.5\%) had HGMs, including atypical meningiomas $(n=10)$ and anaplastic meningiomas $(n=2)$. The subtypes of low-grade meningiomas included meningothelial meningiomas $(n=18)$, fibrous meningiomas $(n=24)$, transitional meningiomas $(n=32)$, psammo- matous meningiomas $(n=3)$, angiomatous meningiomas ( $n=$ $5)$, microcystic meningiomas $(n=2)$, atypical meningiomas ( $n=$ $10)$, and anaplastic meningiomas $(n=2)$. Thirteen of 84 patients with LGMs (15.5\%) and 2 of 12 patients with HGMs (16.6\%) were men. Five HGMs (41.7\%) and 13 LGMs (15.5\%) demonstrated heterogeneous enhancement. Four HGMs (33.3\%) and 7 LGMs $(8.3 \%)$ showed unclear tumor-brain interface. Nine HGMs (75.0\%) and 44 LGMs (52.4\%) were observed with peritumoral edema. Three HGMs (25.0\%) and 9 LGMs (10.7\%) had bone invasion. No significant differences in sex, enhancement pattern, peritumoral edema, and bone invasion were found between HGMs and LGMs ( $P=1.0, .075, .245$, and .351 , respectively). However, there were significant differences in the tumor-brain interface between the 2 groups $(P=.040)$, and the ages of patients with HGMs (60.25 \pm 14.88 years) were slightly older than those of patients with LGMs $(52.37 \pm 10.36$ years $)(P=.022)$. In addition, the median of maximum tumor size was $7.76 \mathrm{~cm}^{2}$ in LGMs (range, $3.64 \sim 15.33 \mathrm{~cm}^{2}$ ) and $8.98 \mathrm{~cm}^{2}$ in HGMs (range, $\left.7.53 \sim 14.55 \mathrm{~cm}^{2}\right)$. The median of maximum peritumoral edema area was $9.22 \mathrm{~cm}^{2}$ in LGMs (range, $3.42 \sim 19.6 \mathrm{~cm}^{2}$ ) and $17.70 \mathrm{~cm}^{2}$ in HGMs (range, $5.82 \sim 22.23 \mathrm{~cm}^{2}$ ). There were no statistically significant differences in maximum tumor size and peritumoral edema area between the 2 groups $(P=.306$ and .200 , respectively).

\section{Comparisons of the Normalized Diffusion Metrics between HGMs and LGMs}

Figure 1 shows the manifestations of high-grade and low-grade meningiomas on conventional images and diffusion maps. Table 1, On-line Table 1, and Fig 2 show the quantitative comparison of differences in normalized diffusion parameters between the 2 meningioma groups. As shown in On-line Table 2, the intraclass correlation coefficients of diffusion metric values were between 0.801 and 0.993 . DKI values, including $\mathrm{MK}, \mathrm{AK}$, and $\mathrm{RK}$, were significantly higher in HGMs than in LGMs $(P \leq .001$ for all). Additionally, MD and ADC were significantly lower in HGMs than in LGMs ( $P=.003$ and .002 , respectively). However, FA did not show a significant difference between the 2 groups $(P=.938)$.

\section{Comparisons of the Diagnostic Efficiency of the Normalized Diffusion Metrics in Differentiating Tumor Grades}

The normalized diffusion metrics were further used for the receiver operating characteristic curve analyses in differentiating meningioma grades (Table 2 and Fig 3). MK exhibited the maximal area under the curve for differentiating HGMs from LGMs. $\mathrm{RK}$ and AK had lower ones, followed by ADC and MD. The optimal cutoff value of MK was 0.99 with a sensitivity of $95.24 \%$, a specificity of $66.67 \%$, a negative predictive value of $95.2 \%$, a positive predictive value of $66.7 \%$, and a Youden index of 0.619 in the diagnosis. Moreover, the area under the curve of MK was significantly higher than that of MD and FA in differentiating LGMs and HGMs $(P<.038$ and $P=.002$, respectively). However, no significant difference between $\mathrm{MK}$ and $\mathrm{ADC}$ was found in terms of area under the curve $(P=.135)$, and these results are shown in On-line Tables 3-5.

The multivariable stepwise logistic regression analysis showed that MK was the only variable that could be used to independently 


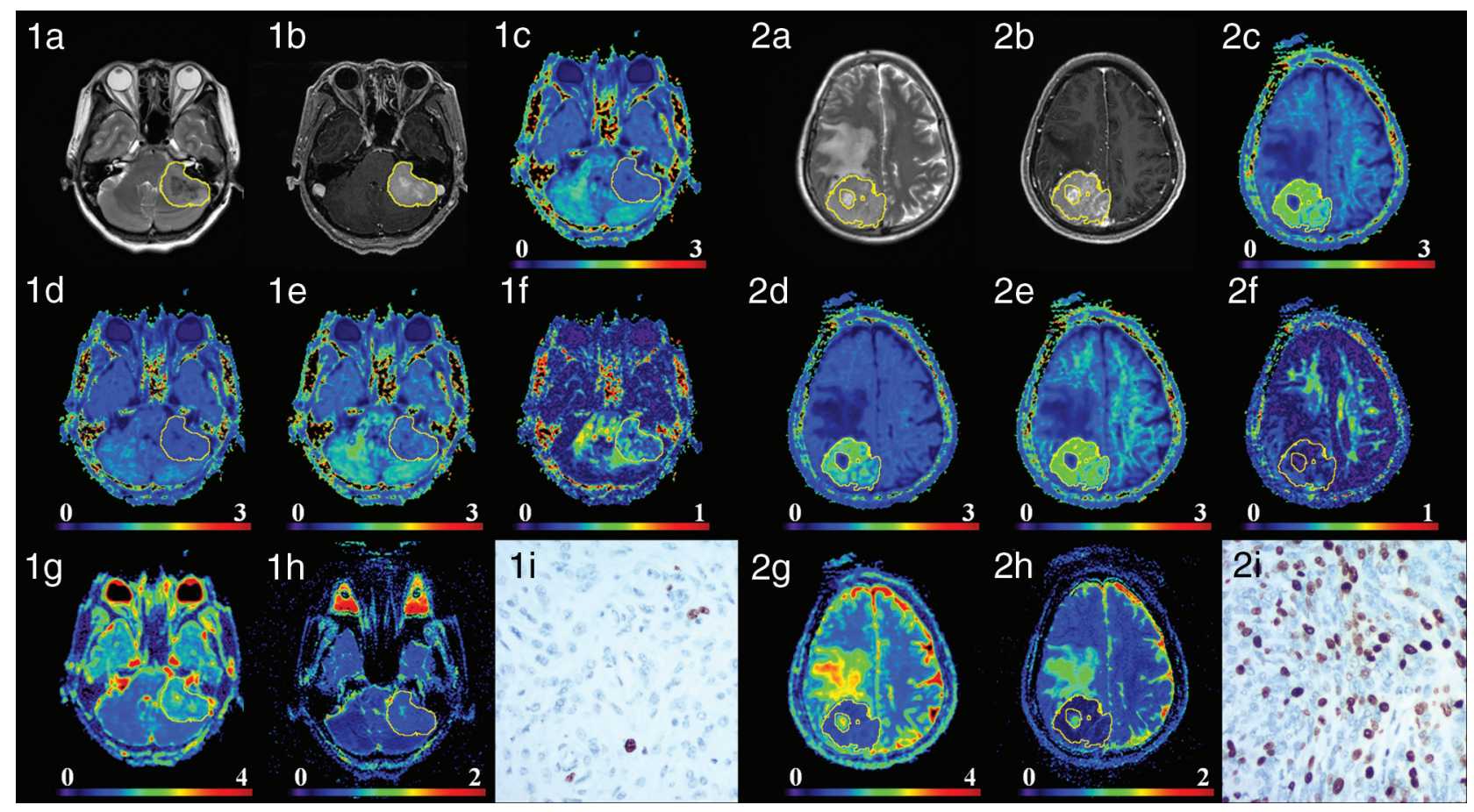

FIG 1. Two patients with low-grade transitional meningiomas in the anterior cranial fossa ( $7 a-7 h)$ and high-grade anaplastic meningiomas in the right cerebral convexity $(2 a-2 h)$. Images $a-i$ are T2Wl, contrast-enhanced T1 FLAIR, MK, AK, RK, FA, MD, ADC, and Ki-67 (100X) images, respectively. For low-grade meningiomas, the intensity was low on MK, AK, and RK maps and high on MD and ADC maps. For high-grade meningiomas, the intensity was high on MK, AK, and RK maps and low on MD and ADC maps. MK, AK, and RK increased, while MD and ADC decreased as the tumor grade increased, whereas FA showed no obvious trend. The Ki-67 values of the 2 patients were $1 \%$ and $30 \%$.

Table 1: Kurtosis and conventional diffusion metric values in the solid region of the tumor normalized by contralateral NAWMa

\begin{tabular}{lcccccc}
\hline Lesion & MK & AK & RK & FA & MD & ADC \\
\hline LGMs & $0.85(0.80 \sim 0.92)$ & $0.93(0.84 \sim 0.99)$ & $0.82(0.72 \sim 0.90)$ & $0.54(0.41 \sim 0.83)$ & $1.18(1.10 \sim 1.30)$ & $1.18(1.08 \sim 1.30)$ \\
HGMs & $1.02(0.91 \sim 1.08)$ & $1.08(0.97 \sim 1.16)$ & $0.99(0.87 \sim 1.05)$ & $0.59(0.41 \sim 0.74)$ & $1.09(0.97 \sim 1.18)$ & $1.03(0.98 \sim 1.15)$ \\
$P$ value & $<.001$ & .001 & $<.001$ & .938 & .003 & .002 \\
\hline
\end{tabular}

Note:-NAWM indicates normal-appearing white matter.

${ }^{a}$ Data are medians, with interquartile ranges in parentheses.

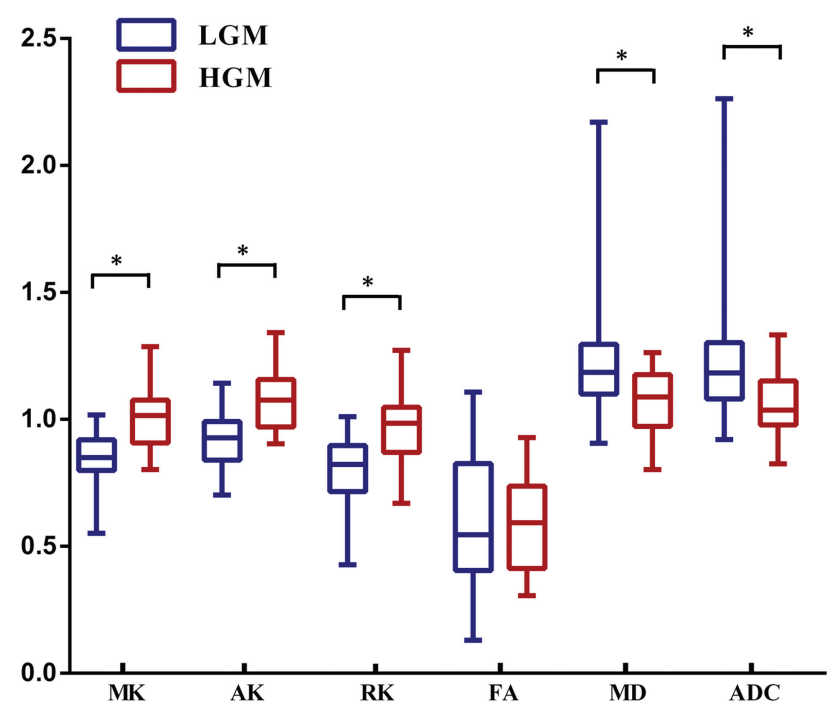

FIG 2. Comparisons of the normalized diffusion metrics between high- and low-grade meningiomas. Lower and upper hinges of boxes denote the 25th and 75th percentiles, respectively. Median (50th percentile) of each distribution is indicated by the line. Whiskers on either side extend to the most extreme data point. Asterisk indicates $P<.01$. differentiate HGMs and LGMs (when MK increases every 0.1, odds ratio $=5.614 ; 95 \%$ confidence interval, $2.150-14.659 ; P<$ $.001)$.

\section{Correlation between Ki-67 and the Normalized Diffusion Metrics}

The level of the proliferation index Ki-67 was available for 59 patients. The difference in Ki-67 between HGMs and LGMs was significant $(12.27 \% \pm 8.10 \%$ versus $2.61 \% \pm 1.69 \%, P<$ $.001)$. Significant correlations were found between Ki-67 and the kurtosis metrics (MK: $r=0.639, P<.001$; AK: $r=0.617$, $P<.001$; RK: $r=0.597, P<.001)$, as well as for MD $(r=$ $-0.369, P=.004)$ and ADC $(r=-0.365, P=.007)$. Corresponding scatter diagrams are shown in Fig 4. The correlation coefficient was maximal for MK and AK, and RK had a lower one, followed by MD and ADC.

\section{DISCUSSION}

An accurate evaluation of the World Health Organization grade and cellular proliferation is particularly important in meningiomas. In this research, our results demonstrated that DKI, DTI, and DWI enabled the differentiation of low-grade from highgrade meningiomas. However, MK obtained from DKI had a 
Table 2: ROC results of all normalized diffusion metrics for differentiating low- from high-grade meningiomas

\begin{tabular}{lllccccc} 
Metrics & AUC & Cutoff & Sensitivity & Specificity & Youden & PPV & NPV \\
\hline MK & 0.842 & 0.99 & 95.24 & 66.67 & 61.91 & 95.2 & 66.7 \\
AK & 0.830 & 0.965 & 66.67 & 83.33 & 50.00 & 96.6 & 26.3 \\
RK & 0.817 & 0.981 & 97.62 & 58.33 & 55.95 & 94.3 & 77.8 \\
FA & 0.493 & 0.743 & 33.33 & 83.33 & 16.66 & 93.3 & 15.2 \\
MD & 0.769 & 1.114 & 71.43 & 75.00 & 46.43 & 95.2 & 27.3 \\
ADC & 0.781 & 1.045 & 89.29 & 58.33 & 47.62 & 93.7 & 43.8 \\
\hline
\end{tabular}

Note:-AUC indicates area under the curve; Cutoff, cutoff value; Youden, Youden index; ROC, receiver operating characteristic; PPV, positive predictive value; NPV, negative predictive value.

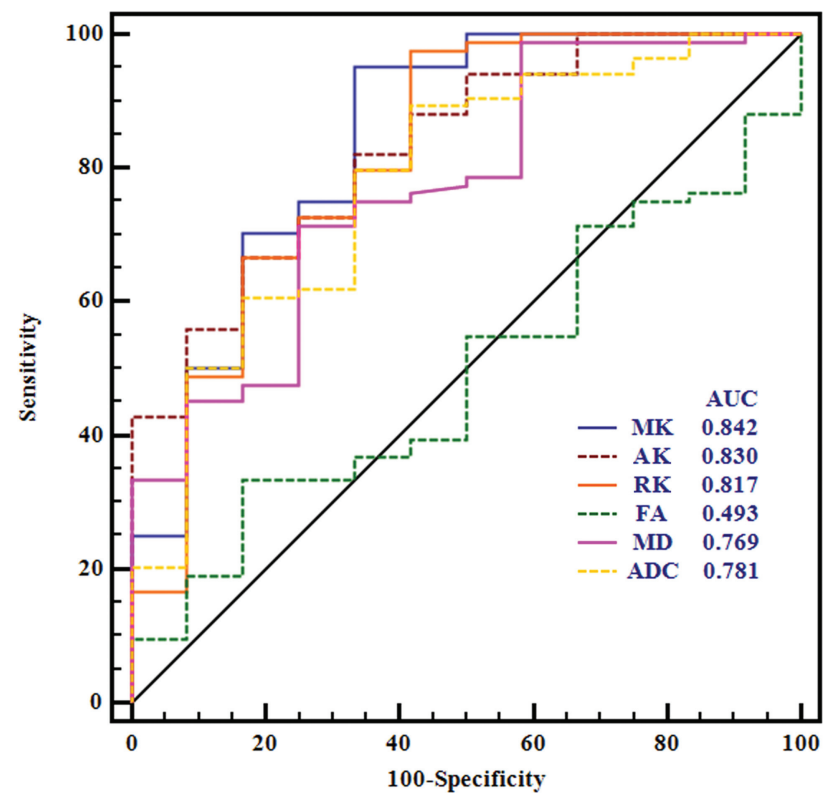

FIG 3. Receiver operating characteristic curves for all normalized diffusion metrics in distinguishing high-from low-grade meningiomas.

greater diagnostic value than DTI metrics in grading meningiomas. MK was the strongest independent predictor associated with the grade of meningioma. In addition, significant correlations were revealed between Ki-67 and the kurtosis metrics, MD and ADC.

There were controversial reports about the value of conventional diffusion imaging in grading meningiomas. Although some studies reported that DTI and DWI can help differentiate malignant from benign meningiomas, ${ }^{12,18-20}$ others suggested that conventional diffusion imaging was not indicative of malignancy grade. $^{8,21}$ In this research, MD and ADC metrics were found effective in the differentiation of HGMs from LGMs, while no significant difference in FA was observed, in line with some previous studies. ${ }^{18,22,23}$ The discrepancies between these studies and our own may be due to the differences in case sizes and inclusion subtypes. In addition, conventional measures of anisotropy using diffusion imaging ignored the interaction between orientation coherence and voxel size, which may cause a strong bias. Recent research indicated that the grade of meningiomas may be better assessed with microscopic anisotropy and microscopic FA. ${ }^{24}$ However, the controversial reports of DTI and DWI may also infer that the reliability of conventional diffusion imaging for grading meningiomas should be explored in a further study. On the contrary, the results of our current study showed that DKI metrics were significantly higher in HGMs than in LGMs; these findings were consistent with those of a previous study. ${ }^{25}$ Pathologically, HGMs were characterized by increased mitotic activity, necrosis, nuclear atypia, and small cells with increased intracellular complex protein molecules and nucleus-to-cytoplasm ratio. ${ }^{8,26}$ As an advanced diffusion imaging sequence, DKI can provide additional kurtosis information, which might characterize the heterogeneity of the microenvironment. ${ }^{16}$ The increase of kurtosis in HGMs probably reflects a higher degree of microstructural complexity within the tumor.

By performing receiver operating characteristic curve analyses, we found that MK obtained from DKI had a significantly better diagnostic performance than $\mathrm{MD}$ and FA derived from DTI. Furthermore, multiple logistic regression analyses revealed that MK was the most significant parameter for grading meningiomas. This is because the diffusion of water molecules in vivo always follows a non-Gaussian distribution. DKI can characterize non-Gaussian water diffusion, while conventional diffusion imaging techniques only assume a Gaussian distribution. Thus, DTI might not be accurate enough to characterize the heterogeneity of tumors. On the contrary, kurtosis is more accurate and sensitive for the detection of microstructural changes, ${ }^{23}$ and MK can indicate microstructural complexity in tumor tissue. ${ }^{11}$ Hence, MK may serve as a more appropriate diffusion metric for assessing and predicting the grade of meningiomas.

In the current study, we further assessed the correlations between diffusion metrics and cellular proliferation of meningiomas. We chose Ki-67 as a biomarker for reflecting tumor cellular proliferation because it is widely used in clinical practice. An elevated Ki-67 proliferation index has been associated with an increased risk of recurrence and poor prognosis in meningiomas. ${ }^{27}$ According to the results, significant positive correlations were revealed between Ki-67 and all kurtosis metrics, while Ki-67 was negatively correlated with $\mathrm{MD}$ and ADC. In malignant tumors, the complexity and heterogeneity of the microenvironment increase and water molecule movement shows restriction both inside and outside of cells. Kurtosis is believed to be generally proportional to the complexity of the microstructure. ${ }^{16,28,29}$ Thus, kurtosis metrics are likely to increase in malignant tumors, while diffusion values are likely to decrease. Meanwhile, the presence of an elevated Ki-67 expression in HGMs indicated an increased mitotic index and cell proliferation. Consequently, cellular proliferation of meningiomas can be noninvasively quantified by diffusion kurtosis metrics. Similar to our observation, a prior study reported the positive correlation between $\mathrm{Ki}-67$ and kurtosis metrics. ${ }^{25}$

The application of diffusion kurtosis metrics in meningiomas is limited. ${ }^{25}$ To our best knowledge, this is the first study to compare DKI with DTI and DWI in grading meningiomas. Our results 
$R^{2}=0.4086, P<0.001$



$R^{2}=0.0004, P=0.884$

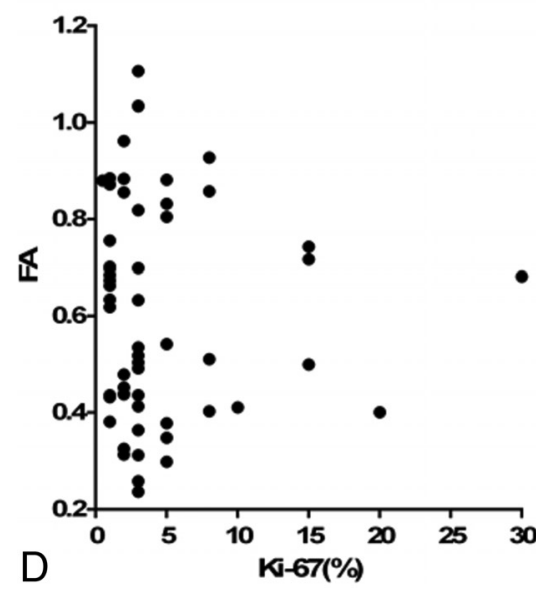

$R^{2}=0.3808, P<0.001$

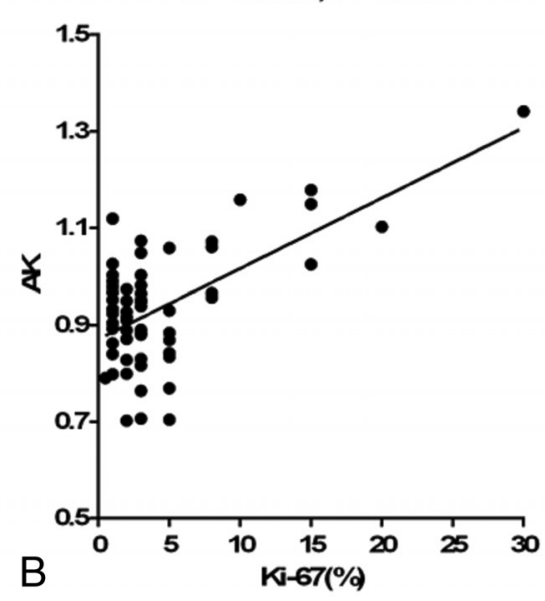

$R^{2}=0.1362, P=0.004$

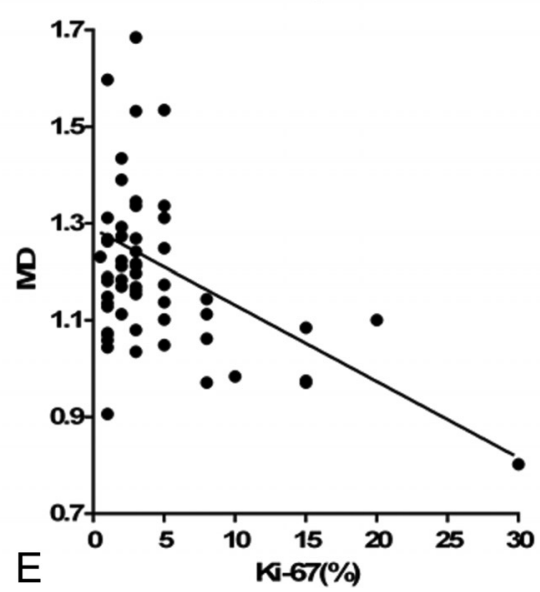

$R^{2}=0.3569, P<0.001$

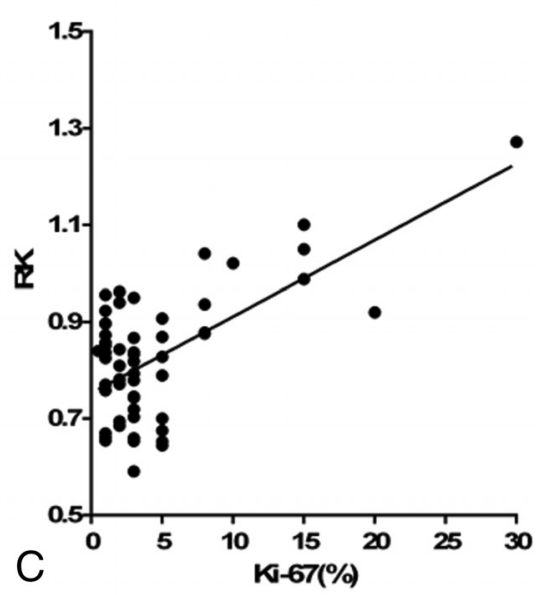

$R^{2}=0.1328, P=0.007$

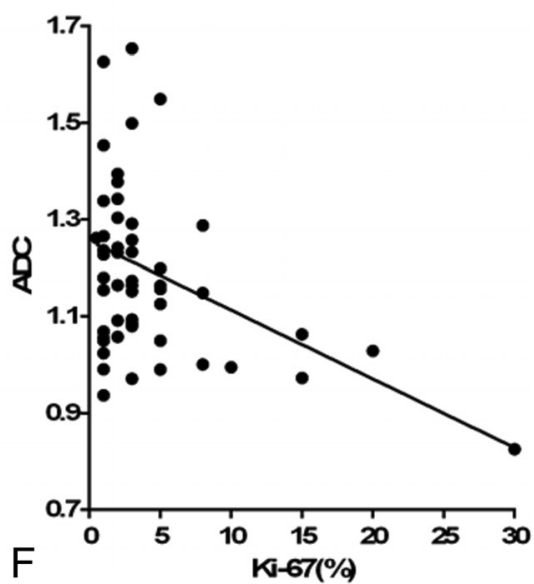

FIG 4. Correlations between Ki-67 and each normalized diffusion metric. Scatter diagrams demonstrate the correlations between the Ki-67 labeling index and MK (A), AK (B), RK (C), FA $(D), \operatorname{MD}(E)$, and $\operatorname{ADC}(F)$.

show that DKI had significantly greater diagnostic properties than DTI in grading meningiomas. In the future, external validation should be performed on a new population to confirm our findings. In addition, a larger, multicenter study population should be explored to obtain meaningful positive and negative predictive values and the real proportions of low- and high-grade meningiomas. We believe that DKI across time may provide clinical practice with robust results and it can consolidate the confidence of clinicians in the grading of meningiomas. Moreover, the promising results found in our study can be partially attributed to the use of a semiautomated method based on threshold segmentation. To date, this is the first study using the semiautomatic method for the measurement of meningiomas. The intraclass correlation analyses showed that the reproducibility of this method was excellent. In addition, it is more reliable, more accurate, and more objective than other methods and has been proposed in our previous studies on gliomas. ${ }^{17}$

This study has several limitations. First, the number of HGMs was relatively limited. A larger sample size may include more cases with HGMs to further verify our results. Second, detailed histologic characteristics such as cell density and nucleoplasmic ratio were not estimated in this study, so the correlation analyses between histologic heterogeneity and diffusion parameters were unavailable. Third, the discordance of some parameters between the DWI and DKI sequences may affect our results. Future studies with consistent parameter settings would help to confirm the findings of the current study. Fourth, the ROI placement of diffusion parameters was not correlated with fragmental histologic specimens, and an MR imagingguided biopsy may be needed to confirm the correlations between diffusion metrics and Ki-67. Fifth, conventional DKI needs relatively long acquisition times, and it may be incompatible with daily clinical practice. A rapid DKI sequence may be beneficial to the clinical use of meningioma grading in further research.

\section{CONCLUSIONS}

Diffusion techniques, including DKI, DTI, and DWI, were useful in grading meningiomas. Moreover, MK demonstrated a better diagnostic efficiency than DTI metrics and was most closely associated with the grade of meningioma. In addition, diffusion metrics showed great potential in reflecting the cellular proliferation of meningiomas.

\section{ACKNOWLEDGMENTS}

The authors thank Zebin Xiao and Zhongping Zhang for their advice on manuscript writing and Ganggang Xu for his assistance with the statistical analyses. 


\section{REFERENCES}

1. Riemenschneider MJ, Perry A, Reifenberger G. Histological classification and molecular genetics of meningiomas. Lancet Neurol 2006; 5:1045-54 CrossRef Medline

2. Louis DN, Perry A, Reifenberger G, et al. The $\mathbf{2 0 1 6}$ World Health Organization Classification of Tumors of the Central Nervous System: a summary. Acta Neuropathol 2016;131:803-20 CrossRef Medline

3. Tang Y, Dundamadappa SK, Thangasamy S, et al. Correlation of apparent diffusion coefficient with Ki-67 proliferation index in grading meningioma. AJR Am J Roentgenol 2014;202:1303-08 CrossRef Medline

4. Abry E, Thomassen IØ, Salvesen ØO, et al. The significance of Ki-67/ MIB-1 labeling index in human meningiomas: a literature study. Pathol Res Pract 2010;206:810-15 CrossRef Medline

5. Lin BJ, Chou KN, Kao HW, et al. Correlation between magnetic resonance imaging grading and pathological grading in meningioma. J Neurosurg 2014;121:1201-08 CrossRef Medline

6. Watanabe Y, Yamasaki F, Kajiwara Y, et al. Preoperative histological grading of meningiomas using apparent diffusion coefficient at $3 \mathrm{~T}$ MRI. Eur J Radiol 2013;82:658-63 CrossRef Medline

7. Yiping L, Kawai S, Jianbo W, et al. Evaluation parameters between intra-voxel incoherent motion and diffusion-weighted imaging in grading and differentiating histological subtypes of meningioma: a prospective pilot study. J Neurol Sci 2017;15 372:60-69 CrossRef Medline

8. Sanverdi SE, Ozgen B, Oguz KK, et al. Is diffusion-weighted imaging useful in grading and differentiating histopathological subtypes of meningiomas? Eur J Radiol 2012;81:2389-95 CrossRef Medline

9. Yamasaki F, Kurisu K, Satoh K, et al. Apparent diffusion coefficient of human brain tumors at MR imaging. Radiology 2005;235:985-91 CrossRef Medline

10. Baskan O, Silav G, Bolukbasi FH, et al. Relation of apparent diffusion coefficient with Ki-67 proliferation index in meningiomas. $\mathrm{Br} \mathrm{J} \mathrm{Ra-}$ diol 2016;89:20140842 CrossRef Medline

11. Van Cauter S, Veraart J, Sijbers J, et al. Gliomas: diffusion kurtosis MR imaging in grading. Radiology 2012;263:492-501 CrossRef Medline

12. Surov A, Gottschling S, Mawrin C, et al. Diffusion-weighted imaging in meningioma: prediction of tumor grade and association with histopathological parameters. Transl Oncol 2015;8:517-23 CrossRef Medline

13. Poot DH, den Dekker AJ, Achten E, et al. Optimal experimental design for diffusion kurtosis imaging. IEEE Trans Med Imaging 2010;29:819-29 CrossRef Medline

14. Tan Y, Wang XC, Zhang H, et al. Differentiation of high-gradeastrocytomas from solitary-brain-metastases: comparing diffusion kurtosis imaging and diffusion tensor imaging. Eur J Radiol 2015; 84:2618-24 CrossRef Medline

15. Pang H, Ren Y, Dang X, et al. Diffusional kurtosis imaging for differentiating between high-grade glioma and primary central ner- vous system lymphoma. J Magn Reson Imaging 2016;44:30-40 CrossRef Medline

16. Tietze A, Hansen MB, Østergaard L, et al. Mean diffusional kurtosis in patients with glioma: initial results with a fast imaging method in a clinical setting. AJNR Am J Neuroradiol 2015;36:1472-78 CrossRef Medline

17. Jiang R, Jiang J, Zhao L, et al. Diffusion kurtosis imaging can efficiently assess the glioma grade and cellular proliferation. Oncotarget 2015;6:42380-93 CrossRef Medline

18. Wang S, Kim S, Zhang Y, et al. Determination of grade and subtype of meningiomas by using histogram analysis of diffusion-tensor imaging metrics. Radiology 2012;262:584-92 CrossRef Medline

19. Toh CH, Castillo M, Wong AM, et al. Differentiation between classic and atypical meningiomas with use of diffusion tensor imaging. AJNR Am J Neuroradiol 2008;29:1630-35 CrossRef Medline

20. Zikou A, Alexiou GA, Goussia A, et al. The role of diffusion tensor imaging and dynamic susceptibility perfusion MRI in the evaluation of meningioma grade and subtype. Clin Neurol Neurosurg 2016; 146:109-15 CrossRef Medline

21. Santelli L, Ramondo G, Della Puppa A, et al. Diffusion-weighted imaging does not predict histological grading in meningiomas. Acta Neurochir (Wien) 2010;152:1315-19; discussion 1319 CrossRef Medline

22. Jolapara M, Kesavadas C, Radhakrishnan VV, et al. Role of diffusion tensor imaging in differentiating subtypes of meningiomas. $\mathrm{J} \mathrm{Neu-}$ roradiol 2010;37:277-83 CrossRef Medline

23. Lu H, Jensen JH, Ramani A, et al. Three-dimensional characterization of non-Gaussian water diffusion in humans using diffusion kurtosis imaging. NMR Biomed 2006;19:236-47 CrossRef Medline

24. Szczepankiewicz F, van Westen D, Englund E, et al. The link between diffusion MRI and tumor heterogeneity: mapping cell eccentricity and density by diffusional variance decomposition (DIVIDE). Neuroimage 2016;142:522-32 CrossRef Medline

25. Xing F, Tu N, Koh TS, et al. MR diffusion kurtosis imaging predicts malignant potential and the histological type of meningioma. Eur J Radiol 2017;95:286-92 CrossRef Medline

26. Nagar VA, Ye JR, Ng WH, et al. Diffusion-weighted MR imaging: diagnosing atypical or malignant meningiomas and detecting tumor dedifferentiation. AJNR Am J Neuroradiol 2008;29:1147-52 CrossRef Medline

27. Modha A, Gutin PH. Diagnosis and treatment of atypical and anaplastic meningiomas: a review. Neurosurgery 2005;57:538-50; discussion 538-50 CrossRef Medline

28. Zhuo J, Xu S, Proctor JL, et al. Diffusion kurtosis as an in vivo imaging marker for reactive astrogliosis in traumatic brain injury. Neuroimage 2012;59:467-77 CrossRef Medline

29. Steven AJ, Zhuo J, Melhem ER. Diffusion kurtosis imaging: an emerging technique for evaluating the microstructural environment of the brain. AJR Am J Roentgenol 2014;202:W26-33 CrossRef Medline 